\title{
ON ENTIRE CONFORMAL MAPPINGS OF SIMPLY CONNECTED REGIONS ${ }^{1}$
}

\author{
ROY W. PENGRA
}

ABSTRACT. Let $\Omega$ be a simply connected proper neighborhood of the origin in the complex plane. Let $\phi$ be a one to one conformal mapping of the unit disk onto $\Omega$, with $\phi(0)=0$. The most general one to one conformal mapping of $\Omega$ onto itself which fixes the origin has the form $f_{\lambda}(z)=\phi\left(\lambda \phi^{-1}(z)\right)$, where $|\lambda|=1$. It is shown that the set of $\lambda$ for which $f_{\lambda}$ is entire and nonlinear has Lebesgue measure zero on the unit circle. The proof depends in part upon properties of solutions, $\phi$, of the functional equation $f(\phi(w))=$ $\phi(\lambda w)$, where $f$ is an entire, nonlinear function, $f(0)=0$ and $|\lambda|=1$.

1. Introduction. Let $\Omega$ be an open, simply connected, proper subset of the complex plane containing the origin. Let $\phi$ be a one to one conformal mapping of the unit disk onto $\Omega$ with $\phi(0)=0$. For each $\lambda \in \mathbf{C},|\lambda|=1$, put

$$
f_{\lambda}(z)=\phi\left(\lambda \phi^{-1}(z)\right) \quad(z \in \Omega) \text {. }
$$

By the uniqueness assertion of the Riemann mapping theorem, $f_{\lambda}$ is the most general one to one conformal mapping of $\Omega$ onto itself which leaves the origin fixed. Our main result, to be proven in $\$ 3$, is

Theorem 1. The set of $\lambda$ for which $f_{\lambda}$ is entire and nonlinear has Lebesgue measure zero on the unit circle.

It is an old conjecture, apparently first made by Kasner [4] in 1912, that the set of $\lambda$ considered in Theorem 1 is in fact empty. Actually, Kasner was interested in the following problem: Given an entire, nonlinear function $f$ with $f(0)=0$ and $f^{\prime}(0)=\lambda$, find a function $\phi$, holomorphic in a neighborhood of the origin, which satisfies

$$
f(\phi(w))=\phi(\lambda w) ; \quad \phi(0)=0, \quad \phi^{\prime}(0) \neq 0 .
$$

It was known as early as $1884[5]$ that (2) always has a solution if $|\lambda| \neq 1$. Kasner [4] conjectured that there are no solutions if $|\lambda|=1$. If $f$ has the form (1), then clearly $\phi$ is a solution of (2). Fatou [2], while studying the sequence

Received by the editors May 10, 1974 .

AMS (MOS) subject classifications (1970). Primary 30A20, 30A64.

1 This research was partially supported by NSF Grant GP-33987X.

Copyright $\odot$ 1975, American Mathematical Society 
of iterates of an entire function, showed that if (2) has a solution $\phi$ with $|\lambda|=1$, then $\phi$ is one to one on its disk of convergence at the origin, and hence $f$ has the form (1). We will give a slightly stronger result in $\$ 2$ needed for the proof of Theorem 1 .

Originally, equation (2) arose in the study of fixed points of holomorphic mappings. Its interest lies in the fact that a solution can be regarded as a change of coordinates making the mapping $f$ linear. It has also been studied in several variables. For an account of the fixed point theory and a fairly complete bibliography we refer the reader to Guckenheimer [3]. For a summary of partial results on the nonexistence of solutions to (2) when $|\lambda|=1$, see Cremer [1].

2. The functional equation. Throughout this section, $f$ will denote an entire, nonlinear function with $f(0)=0$ and $f^{\prime}(0)=\lambda$, where $|\lambda|=1$. For any positive integer $n, f_{n}$ will denote the $n$th iterate of $f$, i.e. $f$ composed with itself $n$ times.

If $\phi$ is a solution of (2), then by applying $f$ to the equation $n$ times, we obtain:

$$
f_{n}(\phi(w))=\phi\left(\lambda^{n} w\right) \quad(n \geq 1)
$$

Suppose $\lambda^{n}=1$ for some $n$. Then (3) would show that $f_{n}(\phi(w))=\phi(w)$. Since $\phi^{\prime}(0) \neq 0$, this would imply that $f_{n}(z)=z$ in some neighborhood of the origin, hence for all $z$, since $f$ is entire. From this it easily follows that $f$ would be one to one and onto the whole plane, contradicting the assumption that $f$ is nonlinear. Thus, equation (2) has no solutions when $\lambda$ is a root of unity.

Theorem 2. Suppose equation (2) has a solution for some $\lambda$, with $|\lambda|=1$. Then $f$ is a one to one mapping of a simply connected set $\Omega$ onto itself, and the solution $\phi$ is a one to one conformal mapping of a bounded disk $D$, centered at the origin, onto $\Omega$. Moreover, $\phi$ cannot be analytically continued across any boundary point of $D$.

Proof. Let $R$ be the (possibly infinite) radius of convergence of the power series expansion of $\phi$ at the origin. Put $D=\{w \in \mathbf{C}:|w|<R\}$.

Let $w_{1}, w_{2} \in D$ and suppose that $\phi\left(w_{1}\right)=\phi\left(w_{2}\right)$. By (3), we then have

$$
\phi\left(\lambda^{n} w_{1}\right)=\phi\left(\lambda^{n} w_{2}\right) \text { for all } n \geq 1
$$

Without loss of generality, assume $\left|w_{1}\right| \leq\left|w_{2}\right|$. Then $w_{1}=\eta w_{2}$ for some 
$\eta \in \mathbf{C},|\eta| \leq 1$. Equation (4) then shows that the functions $\phi(w)$ and $\phi(\eta w)$, both holomorphic on $D$, agree on the set $\left\{\lambda^{n} w_{2}\right\}_{n=1}^{\infty}$. By the remarks above, $\lambda$ is not a root of unity, hence this set has a limit point on $D$, so $\phi(w)=$ $\phi(\eta w)$ for all $w \in D$. The assumption that $\phi^{\prime}(0) \neq 0$ then implies that $\eta=1$ and therefore that $\phi$ is one to one on $D$.

Let $\Omega=\phi(D)$. Then $\Omega$ is a simply connected set, and by (2), $f$ maps $\Omega$ onto itself. Since $f(z)=\phi\left(\lambda \phi^{-1}(z)\right)$ for $z \in \Omega, f$ is also one to one on $\Omega$. If $R=\infty$, then $\phi$ would be an entire, one to one function, and hence be linear. But (2) would then imply that $f$ was also linear, contrary to assumption. Therefore, $R<\infty$.

Suppose $\left|w_{0}\right|=R$, and that $\phi$ can be analytically continued to a neighborhood $N$ of $w_{0}$. Then (3) shows that $f_{n}\left(\phi\left(\lambda^{-n} w\right)\right)$ defines an analytic continuation of $\phi$ in the neighborhood $\lambda^{n} N$ of $\lambda^{n} w_{0}$. Since the sets $\left\{\lambda^{n} N\right\}_{n=1}^{\infty}$ cover the boundary of $D$, this contradicts the fact that $\phi$ must have a singularity on its circle of convergence. Thus, the proof is complete.

3. The main result. Let $\Omega$ be any simply connected neighborhood of the origin in $\mathrm{C}$, other than the whole plane. Let $\phi$ be a one to one conformal mapping from the unit disk onto $\Omega$ with $\phi(0)=0$. For each $\lambda \in \mathbf{C},|\lambda| \leq 1$, put

$$
f_{\lambda}(z)=\phi\left(\lambda \phi^{-1}(z)\right) \quad(z \in \Omega) .
$$

If none of the functions $f_{\lambda},|\lambda|=1$, are entire and nonlinear, then there is nothing to prove in Theorem 1 . So, for the rest of the section, suppose that $f_{\lambda_{0}}$ is entire and nonlinear for some $\lambda_{0},\left|\lambda_{0}\right|=1$.

By Theorem 2, $\phi$ is then one to one on its disk of convergence, $D=$ $\{w \in \mathbf{C}:|w|<R\}$. Let $\Omega^{\prime}=\phi(D)$. Since $1 \leq R<\infty, \Omega \subset \Omega^{\prime}$ and $\Omega^{\prime}$ is not the whole plane. Also, $f_{\lambda}$ is defined and holomorphic on $\Omega^{\prime}$ for all $\lambda,|\lambda| \leq 1$. It follows from (1) that $f_{\lambda}(0)=0$ for all $\lambda$, hence we can write:

$$
f_{\lambda}(z)=\sum_{n=1}^{\infty} c_{n}(\lambda) z^{n},
$$

where the series converges, for each $\lambda$, in every disk centered at the origin which is contained in $\Omega^{\prime}$. Let $r_{0}$ be the radius of the largest disk in $\Omega^{\prime}$ centered at the origin. Since $\Omega^{\prime}$ is not the whole plane, $r_{0}<\infty$. Let $r(\lambda)$ be the radius of convergence of the series (5). Then we have

$$
r(\lambda) \geq r_{0} \text { for all } \lambda,|\lambda| \leq 1 \text {. }
$$

Using this notation, Theorem 1 is an immediate consequence of 
Theorem 1'. $r\left(e^{i \theta}\right)=r_{0}$ a.e. $[d \theta]$.

In order to prove Theorem 1', we will consider the coefficients $c_{n}(\lambda)$ of the series (5). Put

$$
\phi(w)=\sum_{n=1}^{\infty} a_{n} w^{n} \quad(|w|<R)
$$

and

$$
\phi^{-1}(z)=\sum_{n=1}^{\infty} b_{n} z^{n} \quad\left(|z|<r_{0}\right) .
$$

By substituting the power series (7) and (8) into equation (1), we obtain:

$$
c_{n}(\lambda)=\sum_{m=1}^{n} a_{m}\left\{\sum_{\sum_{j=1}^{m} i_{j}=n}\left(b_{i_{1}} \cdots b_{i_{m}}\right)\right\} \lambda^{m} \quad(n \geq 1) .
$$

In particular, $c_{n}(\lambda)$, is a polynomial in $\lambda$ satisfying

$$
c_{n}(0)=0 \quad \text { and } \quad \partial c_{n}(0) / \partial \lambda=a_{1} b_{n} \quad(n \geq 1) .
$$

Lemma 1. There exists a constant $M<\infty$ so that for all $n \geq 1$ and all $\lambda,|\lambda| \leq 1,\left|c_{n}(\lambda)\right|^{1 / n} \leq M$.

Proof. Let $0<R_{1}<R$. Let $D^{\prime}=\left\{w \in \mathrm{C}:|w| \leq R_{1}\right\}$. Since $D^{\prime}$ is a compact subset of $D$, there exists a constant $C<\infty$ so that $|\phi(w)| \leq C$ for all $w \in D^{\prime}$. Let $0<r<r_{0}$ be chosen so that $\{z \in \mathrm{C}:|z| \leq r\} \subseteq \phi\left(D^{\prime}\right)$. Then for all $\lambda,|\lambda| \leq 1$, and all $z,|z| \leq r,\left|f_{\lambda}(z)\right|=\left|\phi\left(\lambda \phi^{-1}(z)\right)\right| \leq C$. Cauchy's inequalities then give $\left|c_{n}(\lambda)\right| \leq C / r^{n}$ for all $n \geq 1$ and all $\lambda,|\lambda| \leq 1$. The lemma now follows by taking $n$th roots.

Lemma 2. Let $\phi$ be holomorphic and one to one on a disk $D$. Let $\Omega=$ $\phi(D)$. If $\phi$ cannot be analytically continued across any boundary point of $D$, then $\phi^{-1}$ cannot be analytically continued across any boundary point of $\Omega$.

Proof. Suppose $\phi^{-1}$ can be analytically continued in some neighborhood $N$ of a point on the boundary of $\Omega$. Since $\phi^{-1}$ is nonconstant, the zeros of the derivative of $\phi^{-1}$ are isolated in $N$. It follows that at some point of the boundary of $\Omega$ in $N, \phi^{-1}$ has a holomorphic inverse. However, this inverse would be an analytic continuation of $\phi$ across some boundary point of $D$, contradicting the assumption.

Proof of Theorem $1^{\prime}$. By $(10), c_{n}(\lambda) / \lambda$ is a polynomial whose value at the origin is $a_{1} b_{n}$. Since $\left|c_{n}(\lambda) / \lambda\right|^{1 / n}$ is subharmonic on $\mathrm{C}$, we have 


$$
\left|a_{1} b_{n}\right|^{1 / n} \leq \frac{1}{2 \pi} \int_{0}^{2 \pi}\left|\frac{c_{n}\left(e^{i \theta}\right)}{e^{i \theta}}\right|^{1 / n} d \theta=\frac{1}{2 \pi} \int_{0}^{2 \pi}\left|c_{n}\left(e^{i \theta}\right)\right|^{1 / n} d \theta .
$$

We therefore obtain,

$$
\begin{aligned}
& \lim \sup _{n}\left|b_{n}\right|^{1 / n}=\lim \sup _{n}\left|a_{1} b_{n}\right|^{1 / n} \leq \lim _{n} \sup _{2 \pi} \frac{1}{2 \pi} \int_{0}^{2 \pi}\left|c_{n}\left(e^{i \theta}\right)\right|^{1 / n} d \theta \\
& \leq \frac{1}{2 \pi} \int_{0}^{2 \pi} \lim \sup _{n}\left|c_{n}\left(e^{i \theta}\right)\right|^{1 / n} d \theta
\end{aligned}
$$

where the last inequality is a consequence of Lemma 1 and Fatou's lemma. By Theorem 2 and Lemma $2, \phi^{-1}$ cannot be analytically continued across any boundary point of $\Omega^{\prime}$. Thus, the radius of convergence of the series (8) is $r_{0}$. The above inequality can therefore be rewritten as

$$
\frac{1}{r_{0}} \leq \frac{1}{2 \pi} \int_{0}^{2 \pi} \frac{1}{r\left(e^{i \theta}\right)} d \theta
$$

However, by (6), $1 / r\left(e^{i \theta}\right) \leq 1 / r_{0}$ for all $\theta$, therefore $r\left(e^{i \theta}\right)=r_{0}$ a.e. $[d \theta]$, completing the proof.

Postscript. Since this paper was submitted, the author has been in correspondence with I. N. Baker who informed him of the following result:

If $f$ is a nonlinear entire function, then the set of entire functions $g$ so that $f(g(z))=g(f(z))$ is countable. (See Math. Z. 79 (1962), 243-249; 104 (1968), 252-256.).

If $|\lambda|=\left|\lambda^{\prime}\right|=1$, then $f_{\lambda}\left(f_{\lambda^{1}}(z)\right)=f_{\lambda^{1}}\left(f_{\lambda}(z)\right)$. It follows that the set considered in Theorem 1 is at most countable.

This result is obtained by considerably different methods than those used in the present paper and the quantitative information in Theorem 1' concerning the radius of convergence of $f_{\lambda}$ does not follow from them.

Professor Baker also informed the author of the work done by C. L. Siegel (Ann. of Math. 43 (1942), 607-612) and by H. Rüssmann (J. Math. Mech. 17 (1967), 523-532) which shows that Kasner's conjecture is false.

\section{REFERENCES}

1. H. Cremer, Über die Schrödersche Funktionalgleichung und das Schwarzsche Eckenabbildungsproblem, Säch. Ges. Wiss. Leipzig Math. Phys. Cl. 84(1932), 291-324.

2. P. Fatou, Sur l'itération des fonctions transcendantes entières, Acta Math. 47 (1920), 337-370.

3. J. Guckenheimer, Endomorphisms of the Riemann sphere, Proc. Sympos. Pure Math., vol. 14, Amer. Math. Soc., Providence, R. I., 1970, pp. 95-123. MR 43 \#500. 
4. E. Kasner, Conformal geometry, Proc. Fifth Internat. Congress Math., vol. 2,1912, p. 83 .

5. G. Königs, Ann. Ecole. Norm 3 (1884), no. 1.

DEPARTMENT OF MATHEMATICS, UNIVERSITY OF WISCONSIN, MADISON, WISCONSIN 53706

Current address: Department of Mathematics, University of Alabama in Huntsville, Huntsville, Alabama 35807

PROCEEDINGS OF THE

AMERICAN MATHEM ATICAL SOCIETY

Volume 50, July 1975

\section{ON QUASINORMAL TOEPLITZ OPERATORS}

\section{ICHIRO AMEMIYA, TAKASHI ITO ${ }^{1}$ AND TIN KIN WONG}

ABSTRACT. It is proved in this note that a quasinormal Toeplitz operator is either normal or analytic.

1. Introduction and the Theorem. Let $L^{p}$ be the Lebesgue space on the unit circle and let $H^{p}$ be the corresponding Hardy space for $1 \leq p \leq+\infty$. The Toeplitz operator $T_{\phi}$ with symbol $\phi$ in $L^{\infty}$ is the operator on $H^{2}$ defined by $T_{\phi} f=P(\phi f)$ for $f \in H^{2}$, where $P$ is the orthogonal projection of $L^{2}$ onto $H^{2}$. A Toeplitz operator $T_{\phi}$ is analytic if its symbol $\phi$ is in $H^{\infty}$. In this case $T_{\phi}$ is simply the multiplication on $H^{2}$ by $\phi$ with the multiplication on $L^{2}$ by $\phi$ as its normal extension. Thus any analytic Toeplitz operator is subnormal. We recall that an operator is subnormal if it is the restriction of some normal operator to one of its invariant subspaces. P. R. Halmos [2] raised the following interesting question: Is every subnormal Toeplitz operator either normal or analytic? The answer is not known yet. Some partial results are given in [3]. As a test question for this problem one may ask if quasinormal Toeplitz operators are either normal or analy-

Received by the editors January 21, 1974 and, in revised form, March 27, 1974. AMS (MOS) subject classifications (1970). Primary 47B20, 47B35.

Key words and phrases. Toeplitz operator, quasinormal operator, subnormal operator.

${ }^{1}$ Research of the second author was partially supported by the National Science Foundation under Grant No. GP-20150. 